\title{
Cases of Swallow Syncope Induced by the Activation of Mechanorecepters in the Lower Esophagus
}

\author{
Ki Hoon Kang, M.D., Wook Hyun Cho², M.D., Myung Chan Kim, M.D., \\ Hee Jong Chang, M.D., Jae II Chung, M.D. and Dong Jun Won, M.D. \\ Division of Cardiology, Department of Internal Medicine, Seoul Adventist Hospital, Seoul, Korea: \\ Division of Cardiology, Department of Internal Medicine, Seoul Paik Hospital, Inje University', Seoul, Korea
}

\begin{abstract}
Swallowing is a rare cause of neurally mediated syncope. The mechanism of swallow syncope that contributes to hypotension, bradycardia, or to both is complex. A 59-year-old man had experienced a recurrent loss of consciousness during swallowing of carbonated beverages or sticky foods. Another 59-year-old man had complained of intermittent syncope just after eating foods. These two patients had no significant structural or functional abnormalities in the esophagus or heart. Both cases showed bradycardia when the lower esophagus was stretched by balloon inflation. The activation of mechanoreceptors in the lower esophagus can be regarded as an initiating factor of these cardioinhibitions. The patients were treated with permanent pacemaker implantation and instructed to change eating habits, respectively.
\end{abstract}

Key Words : Swallow syncope, Mechanoreceptors, Esophagus

\section{INTRODUCTION}

Swallow syncope is a dysautonomic syndrome associated with intense vagal afferent activation due to esophageal stimulation ${ }^{11}$. It causes the loss of consciousness during or shortly after swallowing, due to the reduction of blood flow to the brain through vasodepression and/or arrhythmia. The first report of swallow syncope dates back to 1793 by Spens'; yet, to date there have been only about 50 cases of swallow syncope reported in the literature worldwide. Because of its rarity, the mechanism of swallow syncope could not be understood completely. We report two patients who had swallow syncope without any significant structural or functional abnormalities in the esophagus or heart. We also describe the role of the mechanoreceptors in the lower esophagus that initiate this syncope.

\section{CASE REPORT}

Case 1.

A 59-year-old man was admitted to hospital for the evaluation of syncope. The patient had been in good health except for when hypertension was detected 20 years earlier, and he had subsequently been treated for 3 years. Since he was about 30 years old, he had experienced many episodes of syncope when drinking a carbonated beverage (eg., a soda pop) and beer. About 4 years ago, syncope occurred while drinking cold water when he was taking a bath. Eating sticky foods such as a sweet potato, or even fast running caused syncope. The physical examination and routine laboratory results were normal except for a slightly increased serum creatinine level (132.6 $\mu \mathrm{mol} / \mathrm{L})$. Electrocardiography and chest $\mathrm{X}$-ray findings were not specific. The left ventricular ejection

- Received : April 26, 2004

- Accepted : June 12, 2004

- Correspondence to : Wook Hyun Cho, M.D., Division of Cardiology, Department of Internal Medicine, Seoul Paik Hospital, Inje University, 85, 2 ga, Jeo dong, Jung gu, Seoul, 100-032, Korea Tel : 82-2-2270-0001, Fax : 82-2-2279-4021, E-mail : whcho@inje.ac.kr 
fraction in the echocardiogram was $55 \%$ and concentric left

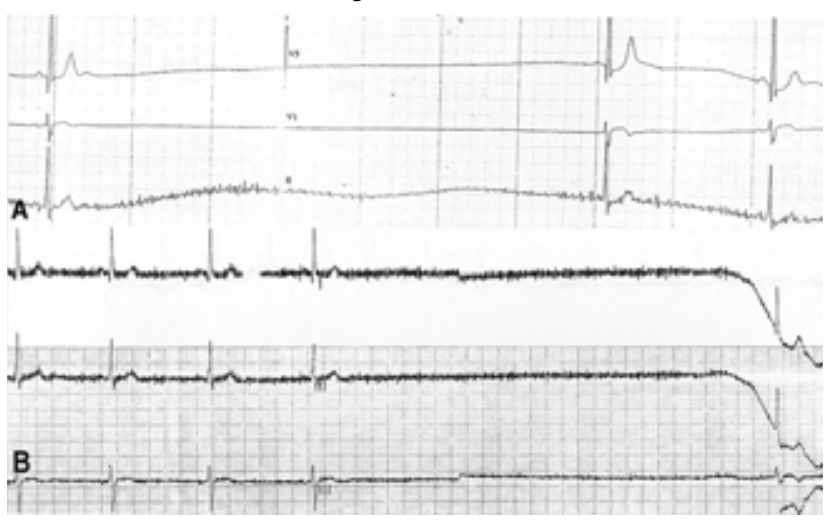

Figure 1. (A) Holter monitoring immediately following the ingestion of a cup of beer (Case 1). The patient had a 7.2-second sinus pause. (B) Electrocardiogram monitoring of the challenge test with a carbonated beverage (a soda pop). The sinus bradycardia caused by the first sip of a soda pop turned into a sinus arrest for 5.4 seconds after the second sip.

ventricular hypertrophy was detected. A brain CT was normal. A carotid massage did not evoke any abnormal rhythms. Holter monitoring showed a sinus arrest with a maximum 7.2 seconds pause while drinking beer and a carbonated beverage (Figure 1A, 1B). An esophagogastroscopy, performed to search for an abnormality in the esophagus and stomach, showed only superficial gastritis at the fundus. Gastric distension by air could not induce syncope or an abnormal rhythm. An esophageal manometry showed no specific esophageal motility disorder except for transient low pressure in the lower portion of the esophagus. The patient was treated with a permanent pacemaker. After backing up the permanent pacemaker, esophageal balloon inflation by a Sengstaken- Blakemore tube with the pressure of $40 \mathrm{mmHg}$ at the lower esophagus induced marked sinus bradycardia (Figure 2). However, no response was observed at the middle and upper esophagus.

\section{Case 2}

A 59-year-old male was referred to hospital for the evaluation of about four episodes of syncope which he had experienced for 1 year. The first episode occurred with a loss of consciousness for 5 minutes following eating foods with feeling obstruction in the esophagus. No specific kinds or forms of foods had been associated with the symptom. He had experienced syncopal episodes usually right after eating, but sometimes while eating foods. The patient had been in good health except for having about ten episodes of an obstructive feeling while eating for 3 years. The result of an esophagogastroscopy conducted 1 year ago had been normal. Physical examination results showed no abnormality. The laboratory result showed that he had Graves' disease (TSH: $0.003 \mathrm{mU} / \mathrm{L}$,

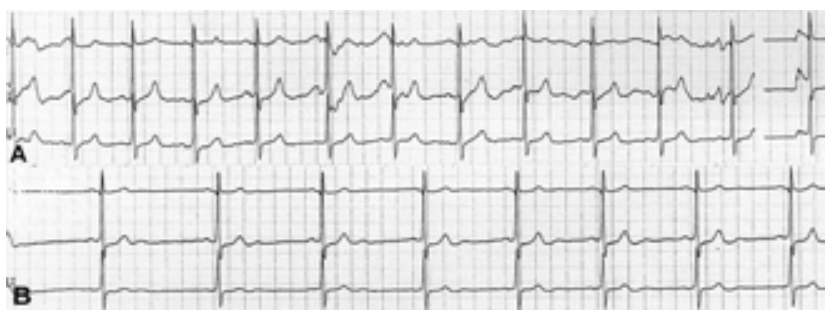

Figure 2. (A) A basal electrocardiography shows normal sinus rhythm (75 beats per minutes) in case 1. B. Esophageal balloon inflation at the lower esophagus initiated marked sinus bradycardia ( 33 beats per minutes) that could not be induced at the middle and upper esophagus.

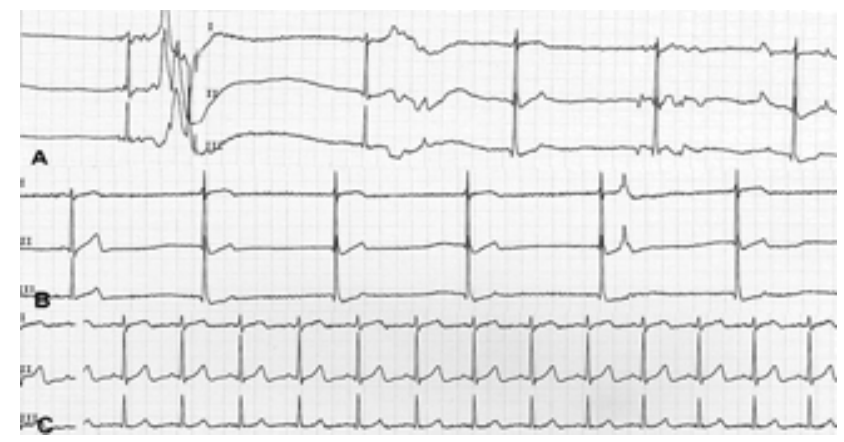

Figure 3. (A) Esophageal balloon inflation at the T7 level of the esophagus made a sinus pause (3.08 seconds) and marked bradycardia. (Case 2) B. Esophageal balloon inflation at the T9 level of the esophagus initiated a junctional rhythm. C. Esophageal balloon inflation at the T9 level of the esophagus after atropine $1 \mathrm{mg}$ IV blocked the reflex.

$\mathrm{T}$ : thoracic vertebra

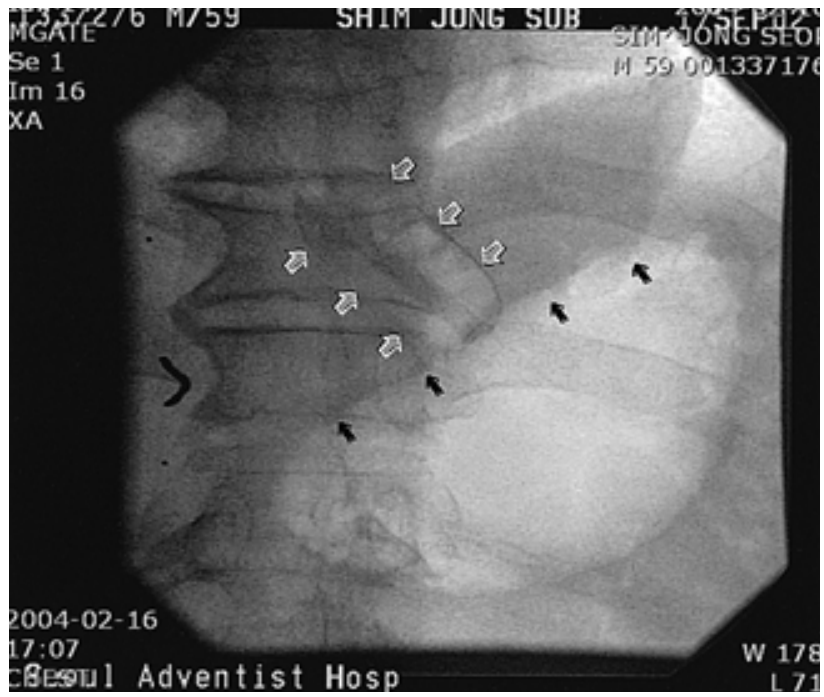

Figure 4. Esophageal balloon inflation done under fluoroscopy. White hollow arrows indicate an esophageal balloon inflated at the lower esophagus (9th thoracic vertebra level in case 2). Black arrows indicate the margin of air shadow in the stomach. 
$\mathrm{FT}_{4}$ : $26.445 \mathrm{pmol} / \mathrm{L}, \mathrm{TSH}$ receptor antibody $30.5 \%$ ). The blood count and liver function tests were normal. An electrocardiography showed sinus bradycardia (57 beats per minutes). A chest $\mathrm{X}$-ray showed a compression fracture at the lower thoracic spine. A thyroid scan showed no demonstrable abnormal finding. The Holter monitoring test could not be done for refusal of patient and rarity of the symptoms. Inflation of the balloon in the lower esophagus and gastroesophageal junction that was at the 9th thoracic spine level resulted in junctional rhythm (32 36 beats per minutes) with lightheadedness (Figure 3, Figure 4). However, an atropine pretreatment blocked this response (Figure 3C). A syncopal episode was considered to occur with eating a bolus of food in a hurry; thus, he was treated by changing his eating habits for syncope and given propylthiouracil for Graves' disease.

\section{DISCUSSION}

According to the analysis of 1,002 syncopal patients, included from 1984 to 1990, the causes of syncope can be classified into three groups: $18 \%$ of cardiogenic, $48 \%$ of non-cardiogenic, and $34 \%$ of unknown causes. Swallow syncope is included in $5 \%$ of the syncopal causes, and the situational syncope syndromes include defecation syncope, micturition syncope, and cough syncope ${ }^{3)}$. People who are middle aged or older have been associated with swallow syncope, yet it may occur in all age groups including children. A 5-year-old girl was reported with a syncopal history during eating since age $2^{4)}$.

\section{Related diseases of swallow syncope}

In Palmer's review, achalasia, diverticulum, esophageal stricture, hiatal hernia, esophageal spasm and cancer are reported as related esophageal diseases ${ }^{11}$. Swallow syncope has occurred also in the setting of cardiac conditions such as an inferior wall myocardial infarction, rhematic myocarditis, and an aortic valve calcification extending into the crest of the intraventricular septum and interrupting the His bundle $e^{1,4,7)}$. Various drugs such as digoxin, $\beta$-blockers (eg., propranolol), calcium channel blockers (eg., verapamil) and ACE inhibitors (eg., enalarpril) have been regarded as aggravating factors ${ }^{6}$. Rarely have illuminating gas inhalation, a diphtheria infection in childhood, metastatic cancer of the bronchus, and surgery of an aortic aneurysm been the causes. Pathologically aberrant communication between vagal afferent and efferent fibers after a trauma, demyelination, mechanical irritation and inappropriate connections of esophageal afferents to medullary cardiovascular interneurons have been reported as causes ${ }^{7}$.

Triggering factors
Syncope may occur by swallowing solid foods, only liquids, or both solids and liquids. The temperature, eating habits and stickiness of foods are associated as the triggering factors. Type of liquids (eg., carbonated beverage) and the presence of belching have been involved with the triggering in some cases $^{4,7,8)}$.

\section{Mechanism}

The mechanism of swallow syncope involves the autonomic nervous system of the esophagus and heart via the vagus nerve ${ }^{7}$. Cardioinhibition caused by inflation of the balloon in the lower esophagus was blocked by the atropine pretreatment in case 2, which implies the involvement of the vagus nerve in the mechanism. The afferent impulses from the esophageal plexus via the vagus nerve travel to the nucleus solitarius tract (NST) at the bran stem. Signals from the NST travel to an adjacent motor nucleus that gives rise to efferent fibers through the vagus nerve to the heart. Thereby, impulses from the esophagus through the reflex arc influence heart rhythm ${ }^{9}$. A variety of arrhythmia has been observed in swallowing syncope. The atrioventricular block has been most often reported; less frequently, sinus bradycardia, a sinoatrial block and a complete atrial and ventricular asystole have been reported. Rarely have sinus tachycardia, supraventricular tachycardia, ectopic atrial tachycardia, paroxismal atrial fibrillation and ventricular arrhythmia been reported ${ }^{1,4,7)}$. Less commonly reported, is when the afferent arm involved in swallow syncope arises from the glossopharyngeal nerve secondary to pharyngeal irritation ${ }^{7)}$.

It has been suggested that vasodepression, in various degrees (mainly or partially), contributes to hypotension in swallow syncope ${ }^{8,10)}$. An 86-year-old female patient who required a Heller's cardiomyotomy for postural hypotension and long- standing achalasia showed a reduction of blood flow to the brain without a change in heart rate after swallowing food ${ }^{10}$. It has been postulated that impulses conveyed to the NST travel to sympathetic preganglionic nuclei in the spinal cord and other nuclei of the brain stem and higher central nervous system. Thus, abnormal depression of sympathetic tone results in hypotension".

Though structural and functional disorders of the esophagus or heart are likely associated with each afferent or efferent system, some cases of swallow syncope with no underlying disorders have also been reported ${ }^{10,11}$. In such a case, an abnormal chemorecepter or mechanorecepter of the esophagus has been suggested ${ }^{10}$. In our two cases, the cardioinhibition by balloon inflation was induced only in the lower portion of the esophagus. This means that the mechanoreceptors in the lower esophagus may play an important role in swallow syncope.

Some humoral factors such as epinephrine and nitric oxide have been recognized as important factors in the mechanism 
of vagally mediated syncope ${ }^{7)}$. Yet, the relationship of swallow syncope with a thyroid hormone excess has not been clarified in the literature. Thus, more study would be necessary in order to say that the thyroid hormone excess seen in case 2 might have influenced the syncopal attacks.

\section{Diagnosis}

In order to diagnose swallow syncope, careful history recording is important to recognize the relationship of swallowing and syncopal or presyncopal symptoms ${ }^{4)}$. The Holter monitoring and provocation tests may reveal a definite correlation between swallowing and syncope ${ }^{11,12)}$. In the case in which vasodepression acts as a dominant contributor, blood pressure monitoring is important for excluding a cardiac effect ${ }^{6)}$. An esophagogastroscopy or barium study can be adopted to detect an underlying disease of the esophagus. An electrocardiography and echocardiogram might help to exclude a cardiac origin. An electroencephalography, brain magnetic resonance imaging or computed tomography can been used for differential diagnosis.

\section{Treatment}

In treating patients with swallow syncope, surgical correction of esophageal pathology has been successful in some cases such as esophageal carcinoma and esophageal stricture ${ }^{12)}$. Of the current medical options, the discontinuation or reduction of aggravating drugs (eg., digoxin, ACE inhibitors etc.) should be first considered ${ }^{6,12)}$. Medications to block vagal conduction such as atropine or propantheline may be another choice. Other medications such as adrenaline, epinephrine or isoprenaline have been used for increasing the ventricular rate directly, ${ }^{52}$. However, no medications have been uniformly successful and most of the drugs have unpleasant side effects that have been poorly tolerated. Avoidance of carbonated fluids or other agents associated with symptoms and the correction of eating habits may be all that is required ${ }^{4}$. Permanent pacemaker insertion has been increasingly employed in recent years for patients with no correctable esophageal pathology, especially for those showing cardioinhibiton upon swallowing ${ }^{12}$.

\section{CONCLUSION}

Swallow syncope with no significant structural or functional abnormality of the esophagus and heart showed a cardioinhibitory response with balloon inflation in the lower esophagus only. Abnormality of the mechanorecepters of the lower esophagus or its abnormal reaction to stimuli may be involved in the mechanism of swallow syncope. The problem can be treated with changing eating habits, or if uncontrolled, with permanent pacemaker implantation.

\section{REFERENCES}

1) Palmer ED. The abnormal upper gastrointestinal vagovagal reflexes that affect the heart. Am J Gastroenterol 66:513-522, 1976

2) Spens T. Medical commentaries. In: Major RH, eds. Classic descriptions of disease. 3rd ed. p. 331, Springfield Illinois, Charles C. Thomas, 1978

3) Linzer M, Yang EH, Estes NA 3rd, Wang P, Vorperian VR, Kapoor WN. Diagnosing syncope: pt 1. value of history, physical examination, and electrocardiography. Ann Intern Med 126:989-996, 1997

4) Farb A, Valenti SA. Swallow syncope. Md Med J 48:151-154, 1999

5) Armstrong PW, McMillan DG, Simon JB. Swallow syncope. Can Med Asso J 132:1281-1284, 1985

6) Deguchi K, Mathias CJ. Continuous haemodynemic monitoring in an unusual case of swallow induced syncope. I Neurol Neurosurg Psychiatry 67:220-222, 1999

7) Levin B, Posner JB. Swallow syncope: report of a case and review of the literature. Neurology 22:1086-1093, 1972

8) Olshansky B. A pepsi challenge. N Engl J Med 340:2006, 1999

9) Benditt DG. Neurally medicated syncopal syndrome: pathophysiological concepts and clinical evaluation. Pacing Clin Electrophysiol 20:572584, 1997

10) Carey BJ, de Caestecker J, Panerai RB. More on deg/utition syncope. N Engl J Med 341:1316-1317, 1999

11) Ausubel K, Gitler B. Swallow syncope in an otherwise healthy young man. Am Heart J 113:831-832, 1987

12) Basker MR, Cooper DK. Oesophageal syncope. Ann R Coll Surg Engl 82:249-253, 2000 